\title{
Wireless Sensor Networks for Industrial Process Monitoring and Control: A Survey
}

\author{
Gang Zhao \\ Digital Technology Laboratory \\ Davis, CA, 95618, United States \\ E-mail: tomlouisville@gmail.com
}

Received: February 6, 2011 Accepted: April 5, 2010 DOI: 10.5296/npa.v3i1.580

\begin{abstract}
This paper provides a survey on implementing wireless sensor network (WSN) technology on industrial process monitoring and control. First, the existing industrial applications are explored, following with a review of the advantages of adopting WSN technology for industrial control. Then, challenging factors influencing the design and acceptance of WSNs in the process control world are outlined, and the state-of-the-art research efforts and industrial solutions are provided corresponding to each factor. Further research issues for the realization and improvement of wireless sensor network technology on process industry are also mentioned.
\end{abstract}

Keywords: Process Monitoring and Control, Quality of Service, Reliability, Topology Control, Wireless Sensor Networks. 


\section{Mll Macrothink}

\section{Introduction}

Wireless sensor network technology has demonstrated a great potential for industrial, commercial, and consumer applications. Specifically, in process monitoring and control, process data such as pressure, humidity, temperature, flow, level, viscosity, density and vibration intensity measurements can be collected through sensing units and transferred wirelessly to a control system for operation and management. Adopting WSNs for process monitoring and control provides great advantages over traditional wired system. As a ubiquitous technology, general issues regarding WSNs have been extensively researched in the academic arena. However, WSN technology is not considered mature enough to be widely implemented in process control applications. Even though wireless transmission of data has been utilized for over ten years in process control applications such as supervisory control and data acquisition (SCADA), industrial WSN products for process monitoring and control are not commercially available until recently due to its specific requirements and challenges.

Researchers have explored the various aspects on applications of WSNs in process industry [1]-[5]. Also, there have been tremendous efforts toward the product research and development in industry. In addition to reviewing the recent research and development achievements, this paper will also analyze special issues for implementing WSN technology on industrial process monitoring and control. The rest of this paper is organized as following. In section II, we will briefly introduce the process control system and discuss the industrial trend and achievements toward WSN applications. In section III, we address the advantages of applying WSNs on industrial process monitoring and control. Section IV presents our efforts to date to address the challenges of using WSNs for process industry. In particular we report the accomplishments in academic research and the progress in industry in regarding to resolving these issues. Finally, further research and development in this field is introduced.

\section{Industrial Applications}

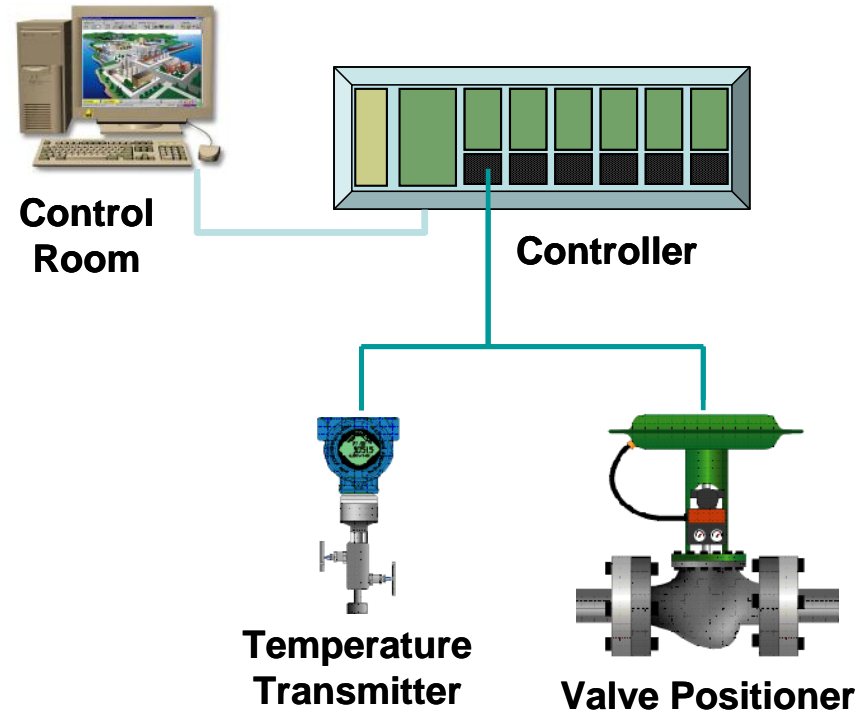

Figure 1. A simple process control system 
Process monitoring and control is a combination of architectures, mechanisms, and algorithms used in the industrial factory for monitoring and control the activities of a specific process to achieve the goal. Let us explain this by illustrating a simple wired application. For example, cooling down a reactor by adjusting the flow rate through the cooling jacket is a process that has the specific, desired outcome to reach: maintaining a constant predefined temperature over time. As shown in Fig. 1, all the devices are hardwired together. Here, the temperature is the controlled variable. At the same time, it is the input variable since it is measured by a temperature sensor and used in a special function to decide the adjustment of a valve to manipulate the flow rate through the cooling jacket. The desired temperature is the setpoint. The valve opening position (e.g. the setting of the valve allowing cooling material to flow through it) is called the manipulated variable since it is subject to control actions. In practice, the temperature value is transmitted to the controller; the controller implements the functions and calculations, transmits the output to control the valve and issues alarm if there are faulty conditions. In the meantime, all data information can be archived for future reference when a review of process trends could provide additional improvements. By utilizing WSN technology, sensing and action devices will communicate wirelessly with an access point (e. g., a gateway or router), which is connected to the control station wirelessly or through wired methods (e.g., Ethernet, Modbus). An example WSN application to replace the wired process control system in Fig. 1 is illustrated in Fig. 2.

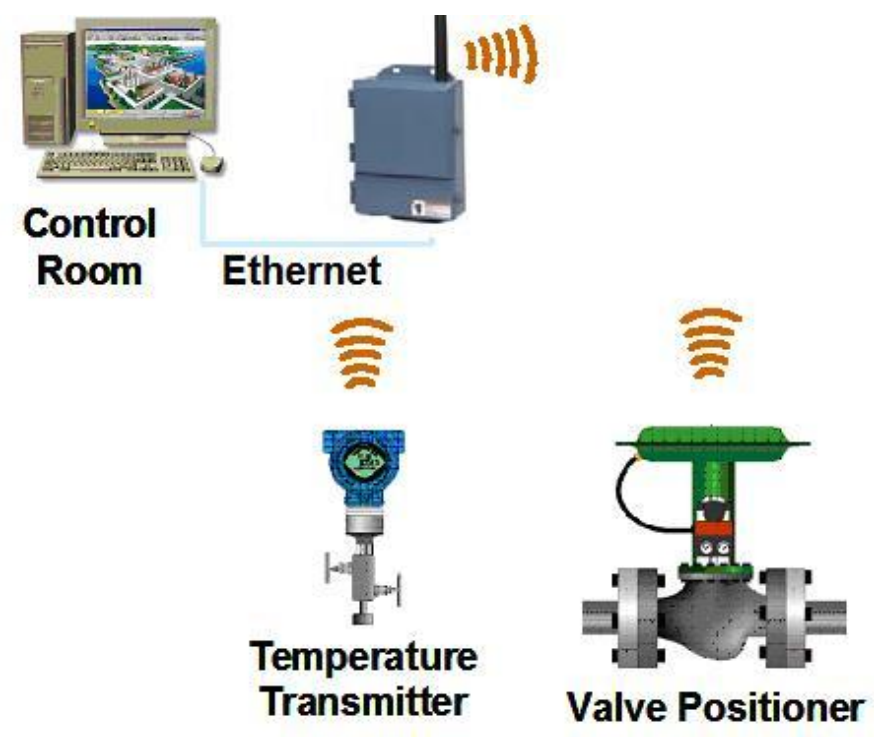

Figure 2. An example WSN for process control

Most of the process control applications are mission critical and have stringent requirements. Failure of a control loop may cause unscheduled plant shutdown or even severe accidents in process-controlled plants. Even when tremendous researches have indicated the compelling benefits of adopting wireless communication technology combined with sensors, a cautious approach was adopted by the technology leaders due to a variety of challenges at the end-user level. The initial applications are mainly focused on process monitoring instead of closed-loop process control. Due to the stringent requirements for closed-loop control, it takes a long journey for the technology transit from wireless process monitoring and 
open-loop control to closed-loop control. It was pointed out in a report [1] on wireless process control products from ISA (International Society of Automation) 2004 that, main stream process control devices such as temperature sensors, valves, and motors that are ready-equipped with wireless transmitters, common network standard and complete wireless process control systems were not yet available. Major achievements have been accomplished recently. WirelessHART (Wireless Highway Addressable Remote Transducer), an open-standard wireless networking technology developed by HART Communication Foundation, was introduced to the market in September 2007. An open-standard wireless networking technology developed by ISA, the ISA100.11a, has also been released on September 2009. Its official description is "Wireless Systems for Industrial Automation: Process Control and Related Applications". Accompanying with these standards, there are emerging wireless process monitoring and control systems together with the wireless transmission enabled main stream process monitoring and control devices. A list of commercial off-the-shelf sensor platforms can be found in [2]. Some example WSN process monitoring and control devices [6]-[9] and their specifications are listed in Table 1.

There have been lots of successful applications all over the world. A wireless self-organizing mesh field network has been applied to monitor wellhead annular pressure and heat exchanger pressures on an offshore platform in the ocean [10]. Even though the wellhead area is crowded with metal obstructions everywhere, remote monitoring is achieved successfully in this harsh, difficult to reach area. Continuous monitoring of pressures eliminates the need for daily visits to the wellhead to manually record gauge readings and enables unusual readings to be identified earlier and action taken to investigate and rectify faults before they develop into serious problems. What's more, the installation took about two hours compared with up to two days for a conventional wired installation. Another exciting application is the temperature monitoring on a rotating drier to ensure that the proper temperature is reached and maintained during the drying process [11]. The cost of wiring for temperature measurement on a rotating drier is prohibitive. With the savings introduced by using wireless transmitters, more temperature transmitters can be located on each drum to increase the amount of process information. Additionally, the five year battery life for each unit lessens the need for periodic transmitter maintenance. 
TABLE I. INDUSTRIAL WSN PRODUCTS

\begin{tabular}{|c|c|c|c|}
\hline Company & Accutech & Honeywell & Emerson \\
\hline $\begin{array}{l}\text { Example } \\
\text { application }\end{array}$ & $\begin{array}{l}\text { Pressure, temperature, } \\
\text { level measurement, } \\
\text { discrete input }\end{array}$ & $\begin{array}{c}\text { Pressure, temperature, level, } \\
\text { position measurement, } \\
\text { discrete input/output }\end{array}$ & $\begin{array}{l}\text { Pressure, temperature, } \\
\text { level, position, vibration } \\
\text { measurement, discrete } \\
\text { input }\end{array}$ \\
\hline $\begin{array}{l}\text { Communication } \\
\text { Technology }\end{array}$ & $\begin{array}{l}\text { Star Point to Point } \\
\text { Communication - Base } \\
\text { station that transmits } \\
\text { and receives data from } \\
\text { multiple field units }\end{array}$ & $\begin{array}{l}\text { Star Mesh Network - The } \\
\text { Honeywell OneWireless } \\
\text { network is formed with } \\
\text { mutli-protocol } \\
\text { communication nodes, called } \\
\text { multinodes, which support } \\
\text { both } 802.11 \text { and field } \\
\text { sensor-based transmissions. }\end{array}$ & $\begin{array}{l}\text { Mesh Network - The } \\
\text { Dust Mesh network with } \\
\text { self healing and self } \\
\text { organization features. }\end{array}$ \\
\hline $\begin{array}{l}\text { Transmission } \\
\text { Technology }\end{array}$ & $\begin{array}{lr}900 \mathrm{MHz} & \text { Frequency } \\
\text { Hopping } & \text { Spread } \\
\text { Spectrum } & \text { (FHSS) }\end{array}$ & $\begin{array}{l}\text { 2.4 GHz Frequency Hopping } \\
\text { Spread Spectrum (FHSS) }\end{array}$ & $\begin{array}{l}\text { 2.4 GHz Direct Sequence } \\
\text { Spread Spectrum (DSSS) }\end{array}$ \\
\hline $\begin{array}{c}\text { Maximum } \\
\text { Transmit/Receive } \\
\text { Range }\end{array}$ & Up to $5000 \mathrm{ft}(\sim 1500 \mathrm{~m})$ & $\begin{array}{l}\text { Up to } 6 \text { miles }(10 \mathrm{~km}) \\
\text { multinode to multimode } \\
\text { communication; sensor to } \\
\text { multinode designed for over } \\
2,000 \mathrm{ft}(600 \mathrm{~m})\end{array}$ & $200 \mathrm{~m}$ \\
\hline $\begin{array}{c}\text { Fastest Update } \\
\text { Time }\end{array}$ & 1 second & 1 second & 4 second \\
\hline $\begin{array}{l}\text { Wireless } \\
\text { Standard }\end{array}$ & $\begin{array}{l}\text { Uses proprietary } \\
\text { protocol }\end{array}$ & ISA $100.11 \mathrm{a}$ & WirelessHart \\
\hline $\begin{array}{l}\text { Number of Field } \\
\text { Units per } \\
\text { Network }\end{array}$ & $\begin{array}{l}\text { Up to } 100 \text { wireless field } \\
\text { units per base radio }\end{array}$ & $\begin{array}{l}\text { Each multinode accepts } \\
\text { signals from up to } 20 \\
\text { wireless transmitters } \\
\text { reporting at } 1 \text { second, and up } \\
\text { to } 400 \text { transmitters reporting } \\
\text { at slower rates. }\end{array}$ & $\begin{array}{l}\text { Up to } 100 \text { devices for a } \\
\text { single wireless gateway }\end{array}$ \\
\hline $\begin{array}{l}\text { Gateway } \\
\text { Interface }\end{array}$ & Modbus & 802.11 Wi-Fi & Ethernet, Modbus \\
\hline
\end{tabular}

\section{Advantages}

WSNs bring several advantages over traditional wired industrial monitoring and control systems as expressed in the following:

\subsection{No Wiring Constraints}

Wireless sensor nodes are installed on industrial devices and equipments to monitor the 
measurements such as proximity, temperature, pressure, level, and power quality, and to transmit/receive control signals for activating the device accordingly. Without the wiring constraints, devices can be utilized in applications that previously are either physically unreachable or cost prohibitive. For example, adopting wireless transmission greatly reduces the complexity of implementing monitoring and control devices for rotary equipments. Furthermore, the industrial process system becomes highly scalable and flexible due to the device autonomy. For example, devices can be easily relocated and reorganized without tedious work on removing old cables and lay outing new ones. In addition, newly added devices can be installed at any location without running power supply and data communication wires through concrete walls during factory expansion.

\subsection{Easy Maintenance}

After the installation of wired device, control engineers have to deal with various wiring maintenance problems such as corrosion, water in the conduit, burned cabling, freezing, wild animal damage, physical wear caused by frequent movement of instrumentation, and unexpected power outage. Wireless device is almost care-free, only a battery change is necessary after years of operation. In addition, it is also possible to relocate current wireless devices or deploy additional wireless devices on the control system after it has been installed with minimal changes to the existing configuration.

\subsection{Reduced Cost}

Cabling and installation for an automation project in an existing facility can run as high as $80 \%$ of total system cost and can exceed $\$ 1,000$ per linear foot in regulated environments, like a typical power plant [12]. Going wireless eliminates the wiring, conduit and installation cost. What's more, for some applications, sensing nodes can put their radio in off mode when necessary, this will save lots of energy compared to wired devices, which requires constant power supply.

\subsection{Better Performance}

Industrial WSNs have the potential to outperform the existing process control network with wired devices. Firstly, it has higher data transmission speed. For example, the most popular control protocols HART (Highway Addressable Remote Transducer) has a data rate of $1.2 \mathrm{kbps}$ and FF (Foundation Fieldbus) has a data rate of $31.25 \mathrm{kbps}$, while WirelessHart has a data rate of $250 \mathrm{kbps}$ based on the IEEE 802.15.4 standard. Secondly, unlike wired control systems, where devices share a single bus, multiple wireless communications can act simultaneously if there is no mutual radio interference [3]. Thirdly, more sensors/datapoints can be used to beat the performance of traditional wired control system.

\section{Challenges and Solutions}

Even though adopting WSN technology to process control systems is attractive, there exists a lot of challenges. The transition from wire to wireless can be beneficial only if related issues are resolved by the combined efforts from both academy and industry. In the following, 
we will address these issues from different perspectives.

\subsection{Quality of Service}

Process monitoring and control applications range from data sensing, measurement, record and diagnosis, to machine/equipment operation and emergency action. These operations are classified by the ISA100 committee into six different classes with increased priority as following [13][14]:

Class 5: Monitoring without immediate operational consequences.

Class 4: Monitoring with short-term operational consequences

Class 3: Open-loop control

Class 2: Closed-loop, supervisory control

Class 1: Closed-loop, regulatory control

Class 0: Emergency action

Data communication in these process control applications includes continuous data request and asymmetric or spontaneous data traffic. For smooth operation, the major concern is the quality of service, which requires the correct data at the right time, i.e., the reliability of the data and the real-time guarantee.

For the reliability of the data, challenges are coming from poor quality radio frequency (RF) links due to high noise, low signal due to lack of power, long path lengths or obstacles, multipath fading, or interference. For example, machines such as vibrator, motors and actuators in the factory can produce a significant amount of electromagnetic noise. Interference or noise in the same frequency band from other sources can corrupt or even eliminate the data signal. The changing radio conditions introduce uncertainties on data packets loss and transmission delay.

The following actions can be taken to improve the accuracy, integrity, and freshness of data.

- Radio transmission technology: For physical layer radio transmission, the Direct Sequence Spread Spectrum (DSSS) or Frequency Hopping Spread Spectrum (FHSS) technology has been utilized to significantly reduce noise interference. DSSS transmits data by spreading out over a wide frequency range. It avoids interference by configuring the spreading function in the receiver to concentrate the desired signal but spread out and dilutes any interfering signal. While FHSS transmits data in a narrow frequency that "hops" around a wide frequency band. It avoids interference that occurs at a particular frequency. Using spread spectrum significantly increases the reliability that the correct data is received even in high noise environments. Also, higher gain or directional antenna can be used to improve signal strength.

- Diversity technique: Different radios may be utilized for operation of various ranges. Also, a sensor's radio transceiver can be capable of changing its transmission power to achieve different transmission ranges.

- Multi-path technique: Signals may be routed/relayed through multiple wireless nodes. In mesh network, multiple paths are used for delivery a single message to guarantee its 
reliability to $99.9 \%$ [15]. For example, if one path is block by obstacle (e. g., a driving by track), the data can still be transmitted through other paths.

- Synchronization and identification technique: Time synchronization techniques are used [16][17] to synchronize all the nodes by time-stamping the data with a global clock. For special application, the process data is also location-stamped [4].

- Evaluation and estimation technique: A WSN self-test technique is introduced to deal with the dynamic RF environment [18]. It indicates that unlike wired channels, where errors come from faulty cables, connector or other hardware components are persistent, transmission errors on wireless channels tend to be transient. By evaluating parameters from a feedback loop, the RF environment characteristics can be estimated and they are used to predict the performance and adapt the operational characteristics of the WSNs to meet the specific application goal. [19] advocates the use of model predictive control (MPC) algorithm to tackle the challenges arises from the usage of wireless technology within feedback control loops. The MPC control algorithm provides an optimized sequence of future control moves based on the process dynamics model, system variable constraints, desired performance specifications, current state and future prediction. Simulation results prove that it can handle certain packet lose in both forward and feed back communication links and it is easy to tune. The drawback is that hybrid MPC is computational intense, so that a time constraint on the optimization solver is necessary to bound the worst-case computation period.

- Redundant technique: Components in WSN can be redundant to increase its reliability, for example, dual gateways are highly recommended for increased reliability. Also, if we can use two or more different frequencies to communicate at the same time, data transmission will continue even if there is interference on one of the frequencies.

Besides reliability requirements, industrial process control also has stringent real-time requirements. As shown in Fig. 1, the real time temperature information is used to decide further operation of the valve positioner. For time critical applications, excessive delay may cause overshoot or disturbance to the process. [20] analyzed the requirements of control system and proposed to use scheduling tool to control the traffic and to limit the retransmission in order to meet this need. To deal with complex control systems that consist of operations of different classes as we introduced before, priority based methods should be adopted to manage the data transmission.

Instead of treating this problem directly, there are also work-around methods. For example, multiple transmission capabilities can be incorporated into a single device. For time critical tasks, devices can switch to high speed mode to transmit message by just one hop. Control in the field can also be used to avoid delay. As shown in Fig. 1, in traditional control systems, information is collected and transmitted to controller; the controller makes the decision and transmits the command to operate the devices. In stead of transmitting signals back and forth to/from controllers, which is usually located remotely, sensing devices can transmit the signals to a highly intelligent device, where the decision is made locally and actions are taken immediately. In the meantime, the results are transmitted to the control room for monitoring and recording. A machine-to-machine application is proposed in [21], 
which claims to cover a wide range of applications. A typical scenario is that a sensor detects an anomaly, sends an alert to a monitoring middleware which in turn generates a command to an actuator, informs a business operation software application, and eventually sends an alarm to an operator off the loop. A method of transmitting directly to control system instead of the controller is also proposed in [3].

In addition to all the above mentioned pro-active preventive or active actions to improve quality of service, post-active countermeasures are also carried out if node failure and package lose arises. Responsive actions must be taken immediately to preclude the failure of certain sensor nodes from affecting the overall task of the sensor network. These actions include implementing fault tolerance methods, formatting new routing path, adjusting of transmission power and sampling rates, commissioning device into fail-safe mode, etc. A novel scheme for detection of faulty sensors is proposed in [22]. In [23], a fault tolerance by quartile method (FTQM) is proposed. In FTQM, the sensing data is firstly divided into four regions. Then, FTQM modifies the region range to select the correct data based on data discreteness. Hence, actors could perform the appropriate actions. [24] proposes to implement a learning and refinement module which enables a fault tolerance solution to be adaptive and self-configurable based on changes in the network conditions.

\subsection{Network Topology}

The network topology is the backbone for any network based systems. When choosing the communication topology for a WSN, in addition to the responsiveness and reliability discussed in Section 4.1, the following aspects need to be considered according to different scenarios: connectivity, adaptability, mobility, and scalability. For example, how to preclude isolated nodes? How does the system adapt to changing RF environments, and loss of key components such as routing nodes and gateways? How does the system support mobile devices such as the handheld field configurator? How does adding a new node affect the latency of existing services in the network?

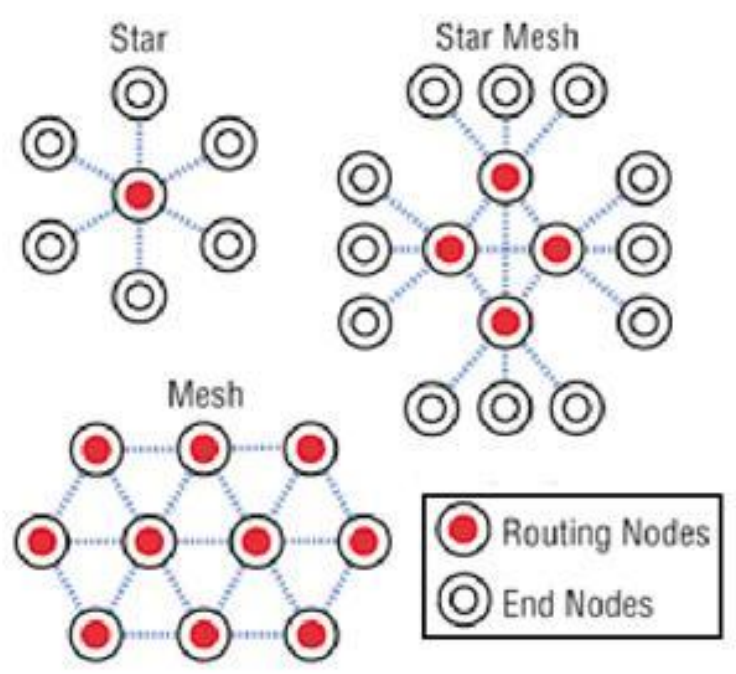

Figure 3. Network Topology 
As shown in Fig. 3, there exist three types of network topology for industrial WSN applications: star, star mesh and mesh. In star network, each node has a designated forwarding path. If there is a failure between two nodes, the information is lost, so that site surveys and link-level configuration are performed during system installation. Compared to star network, star mesh network has increased adaptability, mobility, and scalability by providing multiple routing nodes but they do not offer full end-to-end redundancy. In mesh network, each end nodes are also routing nodes, so that all nodes are fully connected to provide full redundancy. As we discussed in Section 4.1, signals are transmitted from source to sink through multiple paths; if failure happens in one path, the information can still be obtained through other paths. The self organizing and self healing features make the mesh network highly adaptive to node failures/relocation and easily scalable for network expanding. Only for mesh network can a new node be added any where without sophisticated site surveys as long as it is within transmission range of at least two other nodes. It requires less manual configurations for each node and provides greater expandability when more nodes are to be added in the future. This makes it a more suitable choice for deployments that are subjected to changes. The drawback is that extra delay and processing time is introduced due to multiple hops and paths.

\subsection{Resources}

The architecture of a wireless node for process monitoring and control is shown in Fig. 4. For the design of this device, there exist multiple resource limitations, such as processing power and memory limitation, bandwidth limitation, and constrained energy capacity.

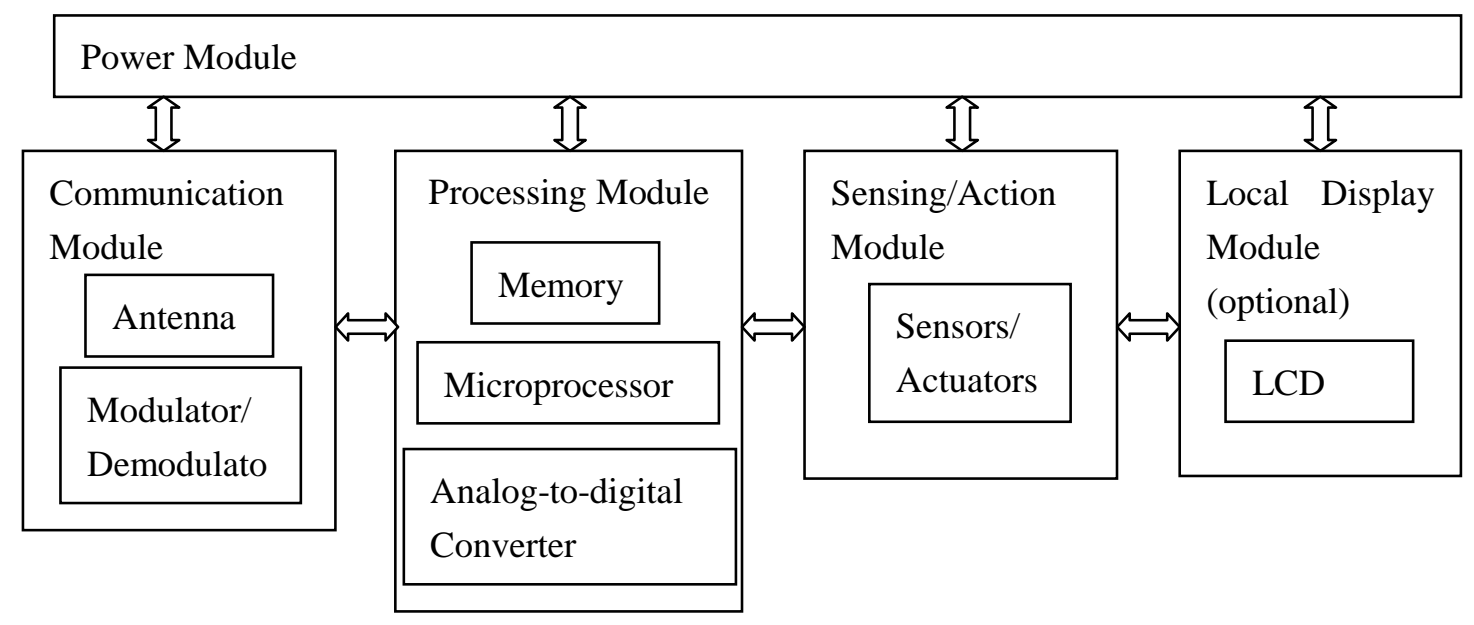

Figure 4. The architecture of a wireless node for process monitoring and control

Hardware limitation: As shown in Fig. 4, the processing module supports the operation system, the network protocol, and handles the data processing and control algorithms. The selection of the microprocessor is critical to the whole design. The ideal microprocessors are those that have large memory and powerful computation capability, consume ultra-low power, and are also energy efficient. An introduction and comparison of available microcontrollers can be found in [25]. [26] provides an interesting heterogeneous multiprocessor sensor node with staged wakeup to keep the device energy efficient. As the technology advances, it is 
promising to have powerful processor and large memory in the sensor nodes with reduced cost.

Bandwidth limitation: Sensor nodes may provide significant redundant data, for example, multiple signals generated from two or more temperature monitoring nodes, or from different sampling period of the same sensing unit. Similar data information can be aggregated by certain functions such as duplicate suppression, minima, maxima, and average to reduce the number of transmissions. Thus, data aggregation is a good technique to achieve required network capacity within the limited bandwidth [5]. By aggregating multiple packets into one packet, the network through will be increased with the reduced overhead for each packet, the drawback is that may introduce extra delay. Coding is also applied in data transmission to increase network capacity [27][28]. Further more, it is mentioned in [29] that instead of transmitting the raw data, only the processed data such as estimation results are transmitted over the WSNs.

Energy limitation: Power consumption remains to be the performance limiting factor and the biggest concern for WSNs. Currently, most industrial applications claim battery life of about five years and WSN systems are not viable in applications that require relatively large amounts of power. Although WSN systems can be applicable in high power applications if a wired power source is run to the devices, it is not preferred or feasible for most applications, where the sensing nodes can only be powered by batteries. A few current solutions are listed as following: 1) Energy efficient transmission: Since fewer data packets and less frequent transmission can save battery power, event-driven energy efficient method aims to reduce power usage by utilizing small data packets and only transmitting when parameters change. Moreover, nodes can operate with a very small duty cycle to conserve power. In other words, they are as inactive as possible for as long as possible. 2) Variable transmission range: Some researchers investigated energy saving by adjusting the transmission range. For example, an energy-balanced consumption scheme by varying the transmission ranges of sensors is proposed in [30]. And an algorithm to search for an optimal transmission range to minimize the total power consumed by the topology management as well as data transmission is introduced in [31]. 3) Energy aware protocols: Energy aware protocols have been explored extensively in the literature. To list a few, a reliable energy aware routing (REAR) protocol for wireless sensor networks is introduced in [32]. REAR considers residual energy capacity of each sensor node in establishing routing paths and supports multi-path routing protocol for reliable data transmission. An energy aware routing protocol for real time traffic in wireless sensor networks is proposed in [33] to consider both energy and delay metric to find an optimal path with minimum energy consumption and minimum end to end delay. 4) Energy harvesting techniques: In addition to battery power, other power technologies such as solar power and energy harvesting from vibrations, blasts of radio can also be used to extend the WSN lifetime [26].

\subsection{Link Layer and Network Layer Protocols}

Medium Access Control (MAC) protocols designed for typical ad hoc networks have primarily focused on optimizing fairness and throughput efficiency, with less emphasis on energy conservation. However, energy constraint is typically considered paramount for 
wireless sensor networks. It has been shown that idle power consumption can be of the same order as the transmit and receive power consumption. The focus of most MAC protocols for sensor networks is to reduce the idle power consumption by setting the sensor radios into a sleep state as often as possible. Sensor-MAC (S-MAC) [40], Timeout-MAC (T-MAC) [41], DMAC [42], TRaffic-Adaptive Medium Access (TRAMA) [43], Sparse Topology and Energy Management (STEM) [44] are a few examples of these protocols.

When designing network protocols for wireless sensor networks, several factors should be considered. First and foremost, because of the scarce energy resources, routing decisions should be guided by some awareness of the energy resources in the network. Furthermore, sensor networks are unique from general ad hoc networks in that communication channels often exist between events and sinks, rather than between individual source nodes and sinks. The sink node(s) are typically more interested in an overall description of the environment, rather than explicit readings from the individual sensor devices. Thus, communication in sensor networks is typically referred to as data-centric, rather than address-centric, and data may be aggregated locally rather than having all raw data sent to the $\operatorname{sink}(\mathrm{s})$. These unique features of sensor networks have implications in the network layer and thus require a re-thinking of protocols for data routing. In addition, sensors often have knowledge of their own location in order to meaningfully assess their data. This location information can be utilized in the network layer for routing purposes. Finally, if a sensor network is well connected (i.e., better than is required to provide communication paths), topology control services should be used in conjunction with the normal routing protocols. Some of the work has been done to address these sensor network-specific issues in the routing layer. These protocols can be classified into three classes: Resource-Aware Routing [45-48], Data-Centric Routing [49], Geographic Routing [50], Clustering for Data Aggregation [51], Querying a Distributed Database [52], Topology Control [53].

\subsection{Security}

Security is also of major concern for WSN applications in process industry. Attacks varies from eavesdropping on transmissions including traffic analysis or disclosure of message contents, to modification, fabrication, and interruption of the transmissions through node capturing, routing attacks, or flooding [2]. When designing the security mechanisms, both low-level (key establishment and trust control, secrecy and authentication, privacy, robustness to communication denial-of-service, secure routing, resilience to node capture) and high-level (secure group management, intrusion detection, secure data aggregation) security primitives should be addressed [34].

It is reported in [21] that this issue is well addressed with existing standards-based technologies. Breaches in security policies are almost technically impossible unless caused by unavoidable issues such as disgruntled employees, theft of access passwords, or bad configuration of the network. AES-128 (Advanced Encryption Standard, with 128-bit keys and 128-bit block size) symmetric-key cryptography algorithm is used in the IEEE 802.11 and IEEE 802.15.4 standards. In WirelessHart, this algorithm is used in conjunction with other security services such as key management (rotating keys for added security), data authentication (for detecting modified data), data freshness service (to avoid replay attacks) 
to provide a complete solution with an equivalent or higher level of security performance than that provided by wired systems. The ZigBee protocol also defines methods for implementing security services such as cryptographic key establishment, key transport, frame protection, and device management [35]. A survey of WSN security can be found in [36].

\subsection{Implementation}

End users are reluctant to choose wireless products due to their concerns on other important issues such as: coverage, interoperability, compatibility, serviceability, longevity, coexistence, availability, delivery and price.

Coverage: For process monitoring, each sensor node obtains information for a limited physical area, so that a given sensor's measurement is limited in both range and accuracy. Careful placement of sensors is required for a full coverage of the field. For the scenario that multiple types of sensors are integrated for monitoring various aspects of a process, and redundant sensing nodes are deployed to tackle adverse environmental conditions such as obstacles and noise, an efficient deployment of sensors is preferred to guarantee the coverage and avoid wasting of sensing units. Here are a few interesting researches in the literature. A grid-based algorithm is proposed in [37]. In this research, the number of the selected sensor nodes is firstly determined based on the probability theory, then an effective selection algorithm is established. Aiming at ensuring successful data delivery, coverage and connectivity in a network for a given lifetime, a novel density-varying deployment scheme for powerful high-end sensors in a heterogeneous sensor network is proposed and optimal placement of high-end sensors whose locations are controllable is studied in [38].

Interoperability: A process monitoring and control project may require products from different suppliers for different functions such as monitoring on temperature, pressure, or level, control for valve, actuator, vibrator, etc. As there are a variety of WSN products available, interoperability is a must-have characteristic. Lots of efforts have been put into standards development and they are recently released to public as we introduced in Section II.

Compatibility: Backward/forward compatibility is a good feature for possible upgrading and performance improvement. Some users may want to make use of existing infrastructure or products, so that product such as adapters from Hart to WirelessHart [54] is developed.

Serviceability: Industrial devices are designed to meet strict requirements to be able to qualify for a variety of hazardous area certifications (flameproof/explosion proof/non-incendive/intrinsically safe). For example, a lot of industrial applications require the product to be intrinsic safe for operation of electronic equipment in explosive atmospheres. This is achieved by ensuring low voltages and currents. Battery powered WSN products are a good fit for these applications.

Longevity: Process control applications range from general purpose application in ordinary locations to special operation in hazardous area under harsh environment. So that devices are engineered to a high level to survive in extremely hot, cold, wet, dirty, abusive, corrosive, and explosive environments. Electronic components are usually encapsulated or fully covered for protection. Devices must undergo various tests such as lifecycle test, 
vibration test, and temperature test before release to market. Due to the tough environment, the hard to reach location and the requirement for the long-term continuous operation, frequent battery change is not recommended. Then energy capability for battery powered devices becomes a major concern. To deal with energy issue, in addition to adopting the techniques in Section 4.2, some products have functions such as remaining energy estimation for better predictability of energy usage.

Coexistence: During implementation, users will also pay attention to coexistence issues to avoid possible interference with existing wireless equipments and to reduce signal interference between WSN nodes.

Availability, delivery and price: WSNs for process monitoring and control are gaining more attention in industry. Some of the potential users may be still waiting to see which standard or product will prevail, but others are already taking actions. Lots of case study for successful WSNs for process monitoring and control applications can be found online [39]. With the increasing market needs, capital investment and mass production, there will be better availability, faster delivery and decreased price for WSN products.

\section{Conclusions}

WSN is a technology with promising future and it is presently used in a wide range of applications to offer significant advantages over wired system. However, WSN technology is not considered mature enough to be widely implemented in process control applications. The demanding constraints for process monitoring and control applications pose many challenges to the implementation of WSNs to the industrial field. In this paper we have surveyed various issues relating to implementing the WSN technology to process monitoring and control.

Future research and development may continue to be focused on further improvements of the reliability and responsiveness, and technology advancements on energy saving, power management, fault tolerance, and smart routing. In addition, software testbed can be developed to evaluate various functions of WSNs such as the self organization, self healing capability. It may also be commercialized as an adds-on software component to evaluate the overall system performance, predict potential problem, and provide suggestions for meeting the desired customer selectable criteria based on the existing system performance. Also, control over wireless is still an emerging research area. The usage of WSN technology within feedback control loops raises lots of challenges to be explored.

\section{References}

[1] D. Chen, M. Nixon, T. Aneweer, R. Shepard, K. Burr, and A. K. Mok, "Wireless process control products from ISA 2004," International Workshop on Wireless and Industrial Automation, March 2005. 
[2] V. C. Gungor and G. P. Hancke, "Industrial wireless sensor networks: Challenges, design principles, and technical approaches," IEEE Trans. Industrial Electronics, vol. 56, no. 10, pp. 4285-4265, October 2009. http://dx.doi.org/10.1109/TIE.2009.2015754

[3] J. Song, A. K. Mok, D. Chen, and M. Nixon, "Challenges of wireless control in process industry," Workshop on Research Directions for Security and Networking in Critical Real-Time and Embedded Systems, April 2006.

[4] M. Antoniou, M. C. Boon, P. N. Green, P. R. Green and T. A. York, "Wireless sensor networks for industrial processes," IEEE Sensors Applications Symposium, February 2009. http://dx.doi.org/ 10.1109/SAS.2009.4801768

[5] M. Hanssmann, S. Rhee, and S. Liu, "No wiring constraints," IEEE Industry Applications Magazine, pp. 60-65, August 2009. http://dx.doi.org/10.1109/MIAS.2009.932593

[6] ISA:

http://www.isa.org/Template.cfm?Section=Standards2\&template=/Ecommerce/ProductDi splay.cfm\&ProductID=10766

[7] Accutech: http://www.accutechinstruments.com/products/

[8] Honeywell: http://hpsweb.honeywell.com/Cultures/en-US/Products/wireless/default.htm

[9] EMERSON:

http://www2.emersonprocess.com/en-US/plantweb/wireless/products/Pages/Wireless-Pro ducts.aspx

[10]EMERSON: http://www.EmersonProcess.com/SmartWireless

[11] Honeywell:

http://hpsweb.honeywell.com/Cultures/en-US/Products/Wireless/Solutions/default.htm

[12]LESMAN Instrument Company:

http://www.lesman.com/unleashd/catalog/wireless/wireless-survey-results.html

[13] ISA: http://www.isa.org/filestore/ISASP100_14_CFP_14Jul06_Final(2).pdf

[14]DUST NETWORKS:

http://www.dustnetworks.com/cms/sites/default/files/SWP_Industrial_Environments.pdf

[15]DUST NETWORKS: http://www.dustnetworks.com/node/203

[16]J. Elson and K. Romer, "Wireless sensor networks: A new Regime for time synchronization," Proceedings of the First Workshop on Hot Topics in Networks (HotNets-I), October 2002. http://dx.doi.org/10.1145/774763.774787

[17]HART Communication Foundation: http://www.hartcomm.org/

[18]P. Jiang, H, Ren, L. Zhang, Z. Wang, and A. Xue, "Reliable application of wireless sensor networks in industrial process control," Proceedings of the 6th World Congress on Intelligent Control and Automation, June 2006. http://dx.doi.org/10.1109/WCICA.2006.1712370

[19]A. Bemporad, S. D. Cairano, E. Henriksson, and K. H. Johansson, "Hybrid model predictive control based on wireless sensor feedback: an experimental study," Proc. IEEE Conference on Decision and Control, pp. 5062-5067, December 2007. http://dx.doi.org/10.1109/CDC.2007.4434918 
[20] J. Song, A. K. Mok, D. Chen, and M. Nixon, "Using real-time logic synthesis tool to achieve process control over wireless sensor networks," IEEE International Conference on Embedded and Real-Time Computing Systems and Applications, August 2006. http://dx.doi.org/10.1109/RTCSA.2006.62

[21] CISCO:

http://www.cisco.com/web/strategy/docs/manufacturing/swpIIWS_Emerson_wp.pdf

[22] T. Banerjee, B. Xie, and D. P. Agrawal, "Achieving fault tolerance in data aggregation in wireless sensor networks,” Global Telecommunications Conference, pp. 926-930, 2007. http://dx.doi.org/10.1109/GLOCOM.2007.178

[23]C. Tuan, Y. Wu, W. Chang and W. Huang, "Fault tolerance by quartile method in wireless sensor and actor networks," International Conference on Complex, Intelligent and Software Intensive Systems, pp. 758-763, 2010. http://dx.doi.org/10.1109/CISIS.2010.12

[24] I. Saleh, H. El-Sayed, and M. Eltoweissy, "A fault tolerance management framework for wireless sensor networks," Innovations in Information Technology, pp. 1-5, 2006. http://dx.doi.org/10.1109/INNOVATIONS.2006.301903

[25] M.A.M. Vieira, C.N., Jr. Coelho, D.C., Jr. da Silva, J.M. da Mata, "Survey on wireless sensor network devices," Proc. Emerging Technologies and Factory Automation, pp. 537-544, September 2003. http://dx.doi.org/10.1109/ETFA.2003.1247753

[26] V. Raghunathan, S. Ganeriwal, and M. Srivastava, "Emerging techniques for long lived wireless sensor networks," IEEE Communications Magazine, vol. 44, no. 4, pp. 108-114, 2006. http://dx.doi.org/10.1109/MCOM.2006.1632657

[27] J. Le, J. C. S. Lui, and D. Chiu, "DCAR: Distributed coding-aware routing in wireless networks," IEEE Transactions on Mobile Computing, vol. 9, no. 4, pp. 596-608, April 2010. http://dx.doi.org/10.1109/TMC.2009.160

[28] S. Katti, H. Rahul, W. Hu, D. Katabi, M. Medard and J. Crowcroft, "XORs in the Air: Practical wireless network coding," IEEE/ACM Transactions on Networking, vol. 16 , no. 3, pp. 497-510, June 2008. http://dx.doi.org/10.1109/TNET.2008.923722

[29]B. Lu, T. G. Habetler, and R. G. Harley, "A novel motor energy monitoring scheme using wireless sensor networks," IEEE Industry Applications Conference, October 2006. http://dx.doi.org/10.1109/IAS.2006.256844

[30]H. Liu, P. Tang, and Z. Erdun, “An energy-balanced variable range transmission scheme in wireless sensor networks," International Conference on Wireless Communications, Networking and Mobile Computing, pp. 1-4, 2009.

[31] J. Shin, M. Chin, and C. Kim, "Optimal transmission range for topology management in wireless sensor networks," Information Networking. Advances in Data Communications and Wireless Networks, vol. 3961, pp.177-185, 2006.

[32] K. Shin, J. Song, J. Kim, M. Yu, and P. S. Mah, "REAR: Reliable energy aware routing protocol for wireless sensor networks," International Conference on Advanced $\begin{array}{llll}\text { Communication } & \text { Technology, } & \text { pp. }\end{array}$ http://dx.doi.org/10.1109/ICACT.2007.358410 
[33]A.H. Mohajerzadeh, M.H. Yaghmaee, "An efficient energy aware routing protocol for real time traffic in wireless sensor networks," International Conference on Ultra Modern $\begin{array}{lllll}\text { Telecommunications } \quad \& \quad \text { Workshops, } & \text { pp. } & 1-9, & 2009 .\end{array}$ http://dx.doi.org/10.1109/ICUMT.2009.5345536

[34]A. Perrig, J. Stankovich, and D. Wagner, "Security in wireless sensor networks," Communication of the ACM, vol. 47, no. 6, pp. 53-57, June 2004. http://dx.doi.org/10.1145/990680.990707

[35]Idaho National Laboratory: http://csrp.inl.gov/Documents/Securing ZigBee Wireless Networks in Process Control System Environments.pdf

[36] J. P. Walters, Z. Liang, W. Shi, V. Chaudhary, "Wireless sensor network security: A survey," in Distributed, Grid, and Pervasive Computing, Yang Xiao Eds. Auerbach Publications, 2006.

[37]W. Li, "Algorithm for wireless sensor nodes placement problem," International Conference on Wireless Communications, Networking and Mobile Computing, pp. 1-3, 2008.

[38]X. Du, X. Liu, and Y. Xiao, "Density-varying high-end sensor placement in heterogeneous wireless sensor networks," IEEE International Conference on Communications, pp.1-6, 2009. http://dx.doi.org/10.1109/ICC.2009.5198894

[39] WINA: http://www.wina.org/pages/default.aspx

[40] Y. Wei, J. Heidemann, and D. Estrin, "An energy-efficient mac protocol for wireless sensor networks," IEEE Conference on Computer and Communications Societies (INFOCOM), 2002. http://dx.doi.org/10.1109/INFCOM.2002.1019408

[41]T. van Dam and K. Langendoen, "An adaptive energy-efficient mac protocol for wireless sensor networks," ACM Conference on Embedded Networked Sensor Systems (SenSys), 2003. http://dx.doi.org/10.1145/958491.958512

[42]G. Lu, B. Krishnamachari, and C. Raghavendra, "An adaptive energy-efficient and low-latency MAC for data gathering in sensor networks," International Workshop on Algorithms for Wireless, Mobile, Ad Hoc and Sensor Networks (WMAN), 2004. http://dx.doi.org/10.1109/IPDPS.2004.1303264

[43] V. Rajendran, K. Obraczka, and J. Garcia-Luna-Aceves, "Energy-efficient, collision-free medium access control for wireless sensor networks," ACM Conference on Embedded Networked Sensor Systems (SenSys), 2003. http://dx.doi.org/10.1145/958491.958513

[44]C. Schurgers, V. Tsiatsis, S. Ganeriwal, and M. Srivastava, "Optimizing sensor networks in the energy-latency-density design space," IEEE Transactions on Mobile Computing, 1(1):70-80, January 2002. http://dx.doi.org/10.1109/TMC.2002.1011060

[45]S. Singh, M. Woo, and C. Raghavendra, "Power-aware routing in mobile ad hoc networks," ACM/IEEE International Conference on Mobile Computing and Networking (MobiCom), 1998. http://dx.doi.org/10.1145/288235.288286

[46] J. Chang and L. Tassiulas, "Energy conserving routing in wireless ad hoc networks," IEEE Conference on Computer and Communications Societies (INFOCOM), 2000. http://dx.doi.org/10.1109/INFCOM.2000.832170 


\section{Macrothink}

[47]R. Shah and J. Rabaey, "Energy aware routing for low energy ad hoc sensor networks," IEEE Wireless Communications and Networking Conference (WCNC), 2002. http://dx.doi.org/10.1109/WCNC.2002.993520

[48]M. Perillo and W. Heinzelman, "DAPR: A protocol for wireless sensor networks utilizing an application-based routing cost," IEEE Wireless Communications and Networking Conference (WCNC), 2004. http://dx.doi.org/10.1109/WCNC.2004.1311672

[49] W. Heinzelman, J. Kulik, and H. Balakrishnan, "Adaptive protocols for information dissemination in wireless sensor networks," ACM/IEEE International Conference on $\begin{array}{llll}\text { Mobile Computing and Networking } 1999 . & \text { (MobiCom), }\end{array}$ http://dx.doi.org/10.1145/313451.313529

[50]G. Zhao, X. Liu, M.-T. Sun and X. Ma, "Energy efficient geographic routing with virtual anchors based on projection distance," Elsevier Computer Communications, vol. 31, pp. 2195-2204, Oct. 2008. http://dx.doi.org/10.1016/j.comcom.2008.02.006

[51]W. Heinzelman, A. Chandrakasan, and H. Balakrishnan, "An application-specific protocol architecture for wireless microsensor networks," IEEE Transactions on Wireless Communications, 1(4):660-670,

October 2002. http://dx.doi.org/10.1109/TWC.2002.804190

[52]P. Bonnet, J. Gehrke, and P. Seshadri, "Querying the physical world," IEEE Personal Communications, 7(5):10-15, October 2000.

[53] Y. Xu, J. Heidemann, and D. Estrin, "Geography-informed energy conservation for ad hoc routing," ACM/IEEE Conference on Mobile Computing and Networking (MobiCom), 2001. http://dx.doi.org/10.1145/381677.381685

[54] MACTek: http://www.mactekcorp.com/

\section{Copyright Disclaimer}

Copyright reserved by the author(s).

This article is an open-access article distributed under the terms and conditions of the Creative Commons Attribution license (http://creativecommons.org/licenses/by/3.0/). 